\title{
LA VOLUNTAD PEDAGÓGICA DE CAJAL, PRESIDENTE DE LA JAE ${ }^{1}$
}

\section{Leoncio López-Ocón Cabrera}

Instituto de Historia-CSIC

\section{RESUMEN}

Este artículo tiene un doble objetivo. Por un lado intenta destacar el papel desempeñado por Santiago Ramón y Cajal en la génesis y desenvolvimiento de la Junta para Ampliación de Estudios e Investigaciones Científicas (JAE). Por otra parte relaciona el protagonismo de Cajal en esa institución con su faceta de educador de los jóvenes científicos. Esa voluntad pedagógica se manifestó en diversos hechos a lo largo de su trayectoria intelectual. Entre ellos cabe destacar su labor de divulgador científico, su afán de participar activamente en la transformación de las estructuras educativas de la sociedad española y su presidencia de la JAE durante un cuarto de siglo, desde 1907 a 1932.

PALABRAS CLAVE: Santiago Ramón y Cajal, educador. Junta para Ampliación de Estudios e Investigaciones Científicas. JAE. 1906. Laboratorios.

\section{THE PEDAGOGICAL WILL OF CAJAL, PRESIDENT OF JAE INSTITUTION} \begin{abstract}
This article has two aims. On one hand, it tries to point out the role of Santiago Ramón y Cajal in the establishment and development of the Junta para Ampliación de Estudios e Investigaciones Cientificas (JAE). On the other hand, it links the leadership of Cajal in this Institution to his teaching authority of young scientifics. This pedagogical will was showed with various facts along his intellectual path. It is appropriate to underline among them his effort in spreading science, his enthusiasm and engagement in order to change the educational structures of Spanish Society and his 25 year long Presidency of JAE, from 1907 until 1932.
\end{abstract}

KEY WORDS: Santiago Ramón y Cajal, teacher. Junta para Ampliación de Estudios e Investigaciones Científicas. JAE. 1906. Laboratories.

1 Una versión preliminar de este trabajo se ha publicado en el número extraordinario de Revista de Educación, coordinado por Antonio Viñao, dedicado al centenario de la JAE, titulado Reformas e innovaciones educativas (España, 1907-1939) 


\section{INTRODUCCIÓN}

Tal y como sostuvo hace tiempo el exiliado Pablo de Azcárate ${ }^{2}$, la Junta para Ampliación de Estudios e Investigaciones Científicas — citada de ahora en adelante por el acrónimo JAE-, creada por real decreto de 11 de enero de 1907, ha sido la «primera obra seria y constructiva de renovación científica, educativa y pedagógica de carácter oficial, realizada dentro del aparato institucional del Estado en la época moderna».

La importancia de la JAE en la historia de la educación y de la ciencia española es pues innegable por tres razones fundamentales, como he planteado en mi Breve historia de la ciencia española ${ }^{3}$.

Fue el principal instrumento que hubo en la España del primer tercio del siglo XX para desarrollar un ambicioso programa de renovación científica y educativa que, aunque se concentró en Madrid, incidió en otras partes del país, y generó la emulación del Institut d'Estudis Catalans, surgido también en 1907 como respuesta cultural y científica del emergente nacionalismo catalán al proyecto de la JAE.

Este organismo público de investigación, pionero en el panorama europeo, pues antecedió a otros organismos similares, como su homólogo alemán la Kaiser Wilhelm Gesellschaft, fundada el 11 de enero de 1911, contribuyó decisivamente a la internacionalización de la ciencia española, gracias al trabajo de sus dos mil pensionados en los principales laboratorios y centros de investigación europeos y americanos.

Y finalmente los científicos de la JAE consiguieron suscitar un interés público por las cuestiones científico-técnicas, como se aprecia, por ejemplo, en el proceso de mitificación que afectó a Santiago Ramón y Cajal (1852-1934) presidente de ese organismo público de investigación desde su fundación en 1907, hasta que cumplió ochenta años en 1932.

La impronta de Cajal en la organización y desarrollo de la JAE es innegable, hasta tal punto de que podría decirse que la JAE contribuyó decisivamente a la «cajalización de España», es decir a poner en práctica el programa educativo y político de Cajal, ya planteado en una de sus primeras obras - Reglas y consejos para la investigación biológica - , de que era posible solucionar los «males de la patria» mediante el cultivo de una cultura de la precisión a través de la

2 AzCÁRATE, P. de (1964), «José Castillejo y la Junta para Ampliación de Estudios», Insula, $\mathrm{n}^{\circ} 209$, p. 6.

3 LóPEZ-OCON, L. (2003), Breve historia de la ciencia española, Madrid, Alianza editorial, pp. 343 y ss. 
investigación experimental. Según Cajal los frutos obtenidos en el trabajo paciente, tenaz y perseverante del laboratorio, locus privilegiado de los científicos, podrían contribuir decisivamente a regenerar el sistema nervioso de una sociedad enferma, convirtiéndose por tanto los lugares en los que se practicaba la ciencia experimental en una especie de sanatorio social. Además Cajal, cuya faceta de educador no ha sido subrayada suficientemente, insistió desde que empezó a tener influencia social en la década de 1890 en que había que impulsar un plan articulado de reformas educativas en la enseñanza superior que sacase de su secular letargo a la Universidad española.

Pero sin embargo ni la abundante bibliografía cajaliana ${ }^{4}$ ni la historiografía existente sobre la $\mathrm{JAE}^{5}$ han insistido suficientemente en la importancia que tuvo Cajal en el desenvolvimiento de esa agencia de promoción de la investigación y de las innovaciones educativas. Se ha tendido a considerar más bien que la Junta para ampliación de estudios e investigaciones educativas fue la obra culmen del institucionismo krausista - tal y como sostuvo ya hace tiempo $\mathrm{M}^{\mathrm{a}}$ Dolores Gómez Molleda ${ }^{6}$ - , y que en su génesis y desarrollo tuvieron un papel protagonista y fundamental la tríada intergeneracional formada por el «abuelo» Francisco Giner de los Ríos (1839-1915), el pedagogo Manuel Bartolomé Cossío (1857-1935), y el catedrático de Derecho Romano y gran administrador y reformador de la educación española el manchego cosmopolita José Castillejo (1877-1945).

Frente a esta opinión mayoritaria historiográficamente quisiera plantear en este texto que la JAE, más bien, surgió gracias a un gran acuerdo entre destacados investigadores experimentales, entre los que sobresalió Cajal, y todo el grupo de intelectuales y científicos krauso-institucionistas, quienes desde

4 Un repertorio bibliográfico sobre la vida y la obra de Cajal en LOPEZ PIÑERO, J.M ${ }^{a}$, Terrada Ferrandis, Ma L., Rodríguez Quiroga, A. (2000), Bibliografia Cajaliana. Ediciones de los escritos de Santiago Ramón y Cajal y estudios sobre su vida y obra, Valencia, Albatros.

5 La obra de conjunto más representativa de esa historiografía es: SANCHEZ RoN, J.M., coord., (1988), 1907-1987. La Junta para Ampliación de Estudios e Investigaciones Científicas 80 años después, Madrid, CSIC, 2 vols. Recientemente han aparecido otros trabajos de conjunto como En el centenario de la Junta para Ampliación de Estudios (1907-2007), $\mathrm{n}^{\mathrm{o}}$ monográfico de Boletín de la Institución Libre de Enseñanza, (2006), II época, $n^{0}$ 63-64; VIÑAO, A. (ed.), (2007), Reformas e innovaciones educativas (España, 1907-1939). En el centenario de la JAE, ${ }^{\circ}$ extraordinario de la Revista de Educación; NARANJO, C. (coord.), La Junta para Ampliación de Estudios y América Latina: memoria, políticas y acción cultural (1907-1939), $\mathrm{n}^{\circ}$ monográfico de la Revista de Indias, (2007), LXVII, no 239.

6 Gómez MolledA, M D D (1966), Los reformadores de la España contemporánea, Madrid, CSIC. 
tiempo atrás habían trabajado para que la moral de la ciencia se convirtiese en moral colectiva dominante en el seno de la sociedad española. Ante el planteamiento que hizo Vicente Cacho Viu ${ }^{7}$, en uno de sus últimos textos, de que las dos corrientes más poderosas del regeneracionismo hispánico, surgido al hilo del desastre del 98 tras la derrota del Estado español frente a la potencia emergente de Estados Unidos, fueron la científica y la nacionalista, impulsadas respectivamente por las elites de Madrid y de Barcelona, y de que el protagonismo de la moral de la ciencia correspondió fundamentalmente a los institucionistas, liderados por Giner, conviene hacer dos matizaciones. Cientificismo y nacionalismo se entrecruzaron en las dos capitales culturales españolas, y un caso ejemplar al respecto es el de Cajal, cuyo afán por alcanzar la gloria científica estuvo impulsado por un patriotismo engendrado en su socialización política en el nacionalismo liberal, que absorbió en su juventud. ${ }^{8} \mathrm{Y}$ evidentemente en el afianzamiento de la moral de la ciencia, que tanta pujanza adquirió en Madrid en el gozne del siglo XIX al XX desempeñó un papel protagonista la labor científica y educativa de Cajal, sorprendentemente ausente en el análisis de Cacho Viu.

Así pues a continuación destacaré el protagonismo de Cajal en la génesis y desenvolvimiento de la JAE resaltando tres hechos, sin minusvalorar por supuesto la aportación krauso institucionista en la puesta en marcha y desarrollo de esa institución.

En primer lugar subrayaré que la JAE se configura en un «annus mirabilis» de la ciencia española, cual es 1906 cuando se le concede el Premio Nobel de Medicina y Fisiología a Santiago Ramón y Cajal.

En segundo lugar enfatizaré que la fundación de la JAE, en enero de 1907, es la culminación de la transformación de la «moral de la ciencia» como moral colectiva dominante o propuesta transformadora de los hábitos educativos y científicos de la sociedad española. En el desenvolvimiento de esa moral de la ciencia trabajó con denuedo Cajal desde la década de 1880 con su trabajo experimental, y con su afán permanente por enseñar a investigar a los jóvenes universitarios, como se aprecia en su discurso de ingreso en la Real Academia

7 CACHO VIU, V. (1997), Repensar el noventa y ocho, Madrid,. Editorial Biblioteca Nueva.

8 Esta cuestión ha sido obviada por importantes historiadores de la ciencia, que sin embargo conocen poco del contexto histórico de la vida y obra de Cajal. Así se aprecia en la crítica hecha por Carlos Solís a la autobiografía de Cajal Recuerdos de mi vida, editada recientemente Juan Fernández Santarén, y publicada por Crítica. El texto de Carlos Solís ha sido publicado en Revista de libros, n 117, septiembre 2006, pp. 3-5. 
de Ciencias Exactas, Físicas y Naturales, de diciembre de 1897, núcleo de su libro Reglas y consejos para la investigación científica, conocido también por su subtítulo de Los tónicos de la voluntad.

Y en tercer lugar señalaré que Cajal, como presidente de la JAE, logró trasladar al funcionamiento del conglomerado de laboratorios de esa institución la misma estrategia que tanta proyección internacional y nacional dio a su propio Laboratorio de Investigaciones Biológicas, creado en 1901, germen, en cierta medida, de la JAE. Esa estrategia consistía en que los investigadores de la JAE debían de prestar atención simultáneamente a una triple labor. Por una parte debían producir o fabricar hechos científicos, mediante el trabajo experimental, usando instrumentos de precisión. Por otro lado tenían que transmitirlos, o favorecer su circulación, entre los pares o colegas, mediante su publicación en relevantes revistas, y/o con una presencia activa en los foros de discusión de los congresos internacionales. Y finalmente debían de esforzarse en diseminarlos en variados escenarios sociales, mediante una labor divulgadora para el gran público y formativa para los educadores científicos.

En todas esas labores Cajal contó con la inestimable ayuda del secretario José Castillejo, con quien formó uno de los dúos más fecundos de la política educativa y científica de la España contemporánea.

Para ambos, como voy a intentar mostrar a continuación, al año 1906, constituyó una especie de «tournant» o viraje decisivo en sus respectivas trayectorias biográficas.

LA IMPORTANCIA DEL AÑO DE 1906 EN LA HISTORIA DE LA EDUCACIÓN Y DE LA CIENCIA ESPAÑOLA

En efecto, la creación de la Junta para ampliación de estudios e investigaciones científicas se gestó a lo largo de 1906 gracias a la convergencia de diversas circunstancias. Por un lado se produjo una oleada de interés público por la ciencia en el seno de la sociedad española, gracias a la concesión a fines de ese mismo año del premio Nobel de Medicina y Fisiología a Santiago Ramón y Cajal, quien lo compartió con el italiano Camilo Golgi. Por otra parte ese premio creó una ventana de oportunidad para que confluyesen los esfuerzos mancomunados del ideario científico y educativo de los institucionistas krausistas, liderados por Francisco Giner de los Ríos y Manuel Bartolomé Cossío, el valor ejemplarizante de la labor investigadora de Cajal, en la cúspide de su prestigio social, y el interés de destacados políticos liberales, como Segismundo Moret (1833-1913), y Amalio Gimeno (1852-1936)), entre otros, por mejorar la instrucción pública y favorecer la renovación científica. 
Todos esos promotores de la JAE, aunque eran conscientes de los efectos benéficos de medidas que se habían tomado años antes para incrementar la internacionalización de la ciencia española, las juzgaban insuficientes. De hecho, el Real Decreto de 18 de julio de 1901, promulgado por el ministerio de Instrucción Pública y Bellas Artes, recién creado, y que establecía una política de envío de pensionados al extranjero para mejorar el nivel intelectual de los profesores universitarios, se puede considerar como un antecedente próximo de la creación de la JAE. En él se afirmaba, en efecto, que «todas cuantas reformas fundamentales se han verificado en nuestra educación nacional proceden de gente que ha vivido en comunicación con el pensamiento científico europeo».

Uno de los integrantes de esas primeras hornadas de pensionados a principios del siglo XX fue un joven José Castillejo. Dado que eran las universidades las encargadas de proponer candidatos, su mentor, Francisco Giner, maniobró entre sus correligionarios institucionistas de la Universidad de Oviedo para que ésta propusiese a su protegido como candidato para perfeccionar sus estudios de Derecho civil en Alemania, lo cual consiguió. Castillejo permanecerá en Berlín y Halle durante el bienio 1903-1905, formándose con prestigiosos juristas - como Rodolfo Stammler9- y observando el funcionamiento de instituciones científicas y centros de enseñanza, que pronto compararía con los británicos, pues en el verano de 1904 se desplazó a Inglaterra. Así pudo comprobar las diferencias entre dos de los mejores sistemas educativos y científicos del mundo en aquella época, fijándose, por ejemplo, en el papel de las mujeres en la vida académica, que era mucho más activo en las universidades inglesas que en las alemanas. De entonces data su admiración del sistema educativo inglés, sobre el que escribiría una importante obra ${ }^{10}$.

Entre tanto, Giner le animó a aspirar a una cátedra. Castillejo escuchó su consejo y obtuvo la cátedra de Derecho Romano de la Universidad de Sevilla a principios de 1905. Ciertamente Castillejo se dedicó con intensidad a esa cátedra solo durante el primer trimestre del curso 1905-1906 pues añorando «aquel Berlín y aquel Halle y esa Institución donde todos los días se encuentra algo nuevo y fresco» ${ }^{11}$ maniobró para regresar lo antes posible a Madrid e in-

9 Ver al respecto CASTillejo, J. (1904 a), «Sobre la enseñanza en la Universidad de Berlín», in Boletín de la Institución Libre de Enseñanza, 28, 267-271; (1904 b): «Un curso de Stammler», in Boletín de la Institución Libre de Enseñanza, 28, 321-329 y 372-377. Artículos citados por Palacios, L. (1979), José Castillejo: última etapa de la Institución Libre de Enseñanza, Madrid, Narcea, p. 35.

10 Castillejo, J. (1919), La educación en Inglaterra, Madrid, La Lectura.

11 Carta de José Castillejo a Francisco Giner, Sevilla, 14 de noviembre de 1905, in CASTILlEJo, D. ed., (1997), Epistolario de José Castillejo, vol. I. Un puente hacia Europa 18961909, Madrid, Editorial Castalia, p. 316. 
corporarse nuevamente a los cenáculos de la Institución Libre de Enseñanza. Su deseo se vio hecho pronto realidad pues una Real Orden de 5 de enero de 1906 le agregó al servicio «de información técnica y de relaciones con el extranjero», negociado del ministerio de Instrucción Pública y Bellas Artes. Desde ahí se convirtió en un instrumento eficaz de los planes educativos y científicos de sus maestros institucionistas, particularmente de D. Francisco Giner de los Ríos, y se entrenó en las funciones de coordinación científico-técnica que posteriormente tan eficazmente llevaría a cabo como secretario de la JAE.

En efecto ese servicio le permitió disponer de una atalaya privilegiada para hacer un seguimiento del movimiento científico español del momento, y detectar a los investigadores más dinámicos, quienes a su vez encontraron en Castillejo a un interlocutor idóneo para satisfacer sus necesidades de información y de comunicación con el movimiento científico europeo. Se aprecia este hecho en la correspondencia que intercambió Castillejo con el prestigioso arabista Asín Palacios, y el historiador Rafael Altamira, cuando ambos estaban embarcados en el lanzamiento de Cultura española, una de las mejores revistas del positivismo historiográfico efectuado en este país, y digna heredera de la publicación promovida años atrás por Rafael Altamira titulada Revista crítica de Historia y Literatura Españolas, Portuguesas e Hispano-Americanas ${ }^{12}$.

Así pues en esos meses de 1906 en los que Castillejo se hizo cargo del servicio de información técnica y de relaciones con el extranjero del ministerio de Instrucción Pública y Bellas Artes trabó relaciones estrechas de colaboración y tejió redes de comunicación científica con diversos colegas inquietos por impulsar y profesionalizar las investigaciones históricas. Precisamente varios de ellos, como los mencionados Miguel Asín y Rafael Altamira, se integrarían años después en los laboratorios de investigación del Centro de Estudios Históricos, cuando la JAE decidió crear esa institución en 1910 para renovar la historiografía española. Pero también durante su permanencia en ese negociado del Ministerio de Instrucción Pública y Bellas Artes Castillejo se hizo cargo del régimen de pensiones que concedía ese ministerio, a propuesta de las universidades. Y tomó conciencia de sus deficiencias e insuficiencias, pues se concedía con cuentagotas. De modo que los pensionados españoles en el extranjero ocupaban el puesto antepenúltimo en el ranking de estudiantes foráneos matriculados en las principales universidades europeas.

Entre tanto en círculos ministeriales de diversos gabinetes liberales se discutía la forma de propiciar un salto cualitativo en el sistema científico espa-

12 Carta de Miguel Asín Palacios a José Castillejo, Madrid, 15 de febrero de 1906, en Castillejo, D. (1997), p. 319; Carta de Rafael Altamira a José Castillejo, Oviedo, 18 de marzo 1906, en CASTILLEJO, D. (1987), p. 321. 
ñol, que ya disponía, al fin, de una lumbrera de renombre internacional representada por Cajal, el cual — como es sabido - había obtenido en 1904 la prestigiosísima medalla Helmholtz, otorgada por la Preussische Akademie der Wissenschaften (hoy Academia de Ciencias de Berlin-Brandenburgo). De hecho, en marzo de 1906 Segismundo Moret, líder en aquel momento de la más importante agrupación liberal, intentó convencer a Cajal para que aceptase la cartera de Instrucción Pública con vistas a realizar un ambicioso plan de reformas educativas, a imitación de las acciones reformadoras llevadas a efecto en la Francia de la Tercera República por el renombrado químico Marcellin Berthelot. Tras diversas vacilaciones, Cajal rechazó la oferta, pero transmitió a Moret un detallado plan para «desperezar la Universidad española de su secular letargo», cuyas principales medidas eran: «la contrata, por varios años, de eminentes investigadores extranjeros; el pensionado, en los grandes focos científicos en Europa, de lo más lucido de nuestra juventud intelectual, al objeto de formar el vivero del futuro magisterio; la creación de grandes colegios, adscritos a institutos y universidades, con decoroso internado, juegos higiénicos, celosos instructores y demás excelencias de los similares establecimientos ingleses; la fundación, en pequeño y por vía de ensayo, de una especie de Colegio de Francia, o centro de alta investigación, donde trabajara holgadamente lo más eminente de nuestro profesorado y lo más aventajado de los pensionados regresados del extranjero; la creación de premios pecuniarios a favor de los catedráticos celosos de la enseñanza o autores de importantes descubrimientos científicos, a fin de contrarrestar los efectos sedantes y desalentadores del escalafón, etc.» ${ }^{13}$

Muy poco después, a principios de junio, la otra gran figura intelectual que estaba desde hacía tiempo abogando por transformar a la moral de la ciencia en moral colectiva dominante, cual era el caso del prestigioso pedagogo institucionistas Francisco Giner, se dirigió a Moret para, tras recordarle los lazos que les unían desde los tiempos en los que ambos fueron discípulos del líder del krausismo español -D. Julián Sanz del Río-, enviarle un memorando. En él se planteaba la nueva estrategia de los krauso institucionistas: abordar la renovación educativa y científica de la sociedad española desde arriba, es decir contando con el impulso del Estado, tras haberlo hecho desde el seno de la sociedad civil durante tres décadas, desde que se creara la Institución Libre de Enseñanza en 1876. Así en ese memorandum Giner instaba a su amigo Moret a preparar una nueva elite dirigente lo más rápida e intensivamente

13 RAmón y CAJAL, S. (1981), Recuerdos de mi vida: historia de mi labor cientifica, Madrid, Alianza Universidad, pp. 286-287. 
posible, y a resolver en el terreno educativo ciertos problemas apremiantes. Para obtener esos objetivos consideraba imprescindible un aumento considerable de las pensiones en el extranjero, así como la adopción de medidas encaminadas a la mejora de la investigación y la enseñanza experimentales mediante la creación de un organismo técnico suprapartidario ${ }^{14}$.

Aunque el gabinete de Moret fue efímero, en las semanas siguientes se mantuvo el acuerdo entre los responsables políticos liberales y los científicos e intelectuales — como Cajal y el trío formado por Giner, Cossio y Castillejo- para conseguir que el cultivo de la ciencia se convirtiese en una moral colectiva dominante en el seno de la sociedad española, como se aprecia en la correspondencia de Castillejo. En efecto en sus cartas del segundo semestre de 1906 Castillejo informa a Giner y Cossío de todas las gestiones realizadas con el Subsecretario del Ministerio de Instrucción Pública, - presidido por el catedrático de Patología de la Universidad de Madrid, y amigo y antiguo compañero de Cajal en la Universidad de Valencia, Amalio Gimeno-, para estudiar los fondos procedentes de funciones benéficas y pedagógicas que pudiesen servir de base para organizar una Junta autónoma, así como de las medidas que había que adoptar para el mejor aprovechamiento de las pensiones y favorecer las relaciones de los pensionados con los centros docentes ${ }^{15}$. Y más adelante señala que en octubre había enviado al subsecretario del ministerio «el preámbulo y las notas que deseaba» ${ }^{16}$. Semanas después, estando en Ciudad Real, comunica a Giner que «al ver aprobados los presupuestos escribo al subsecretario rogándole me avise antes de entregar el Real Decreto de pensiones para revisarlo juntos» ${ }^{17}$.

De manera que, tal y como han destacado Ramón Carande ${ }^{18}$ y Luis Pala$\operatorname{cios}^{19}$, la redacción del Real Decreto que creó la Junta para ampliación de estudios e investigaciones científicas, publicado el 11 de enero de 1907, fue obra directa y personal de José Castillejo, quien ya empezó a ejercer como secretario el día de la constitución de esa institución. Así, el 15 de enero de 1907 redactó como tal secretario el acta de esa reunión en la que los asistentes

14 El borrador de este documento, fechado en Madrid el 6 de junio de 1906, ha sido publicado en CASTILLEJO, D. ed., (1997), pp. 326-329.

15 Carta de Castillejo a Cossío, Madrid, 13 de julio 1906, en ibidem, pp. 331-332.

16 Carta de Castillejo a Giner, Sancti-Spiritus, 17 noviembre 1906, en ibidem, p. 340.

17 Carta de Castillejo a Giner, Ciudad Real 30 diciembre 1906, en ibidem, p. 342

18 CARANDE, R. (1966), «Un vástago tardío de la 'Ilustración': José Castillejo (18771945), in Mélanges á la mémoire de Jean Sarrailh, Paris, Centre de recherches de l'Institut d'études hispaniques, 1, pp. 191-210.

19 Palacios Bañuelos, L. (1979), José Castillejo: última etapa de la Institución Libre de Enseñanza, Madrid, Narcea. 
—Ramón y Cajal, Sorolla, Santa María de Paredes, San Martín, Calleja, Vincenti, Simarro, Bolívar, Menéndez Pidal, Casares, Álvarez Buylla, Rodríguez Carracido, Ribera, Torres Quevedo, Fernández Ascarza, y Castillejo- eligieron por unanimidad presidente de la flamante Junta para Ampliación de Estudios e Investigaciones Científicas a Santiago Ramón y Cajal, el cual, tras regresar de Estocolmo adonde se había dirigido para recibir el premio Nobel que le acababan de conceder, se encontraba en el cenit de su prestigio científico e influencia social. Desde la presidencia de la JAE Cajal pudo en cierta medida impulsar un programa educativo y científico regeneracionista en el que venía trabajando desde tiempo atrás, como explicaré a continuación. Y al parecer su quehacer influyó en el día a día de la administración de la JAE, y en la política científica impulsada por Castillejo, según se deduce de la carta que éste escribiera al ministro liberal Santiago Alba el 31 de octubre de 1912, cuya relación ha sido analizada recientemente por José Manuel Sánchez Ron $^{20}$ (2006), y en la que el secretario de la JAE afirmaba lo siguiente:

Mi querido amigo: Olvidé la otra noche decir a Ud. que me parece será muy bien recibido un aumento considerable para pensiones en el extranjero.

Sólo que, en vez de dárselo a la Junta, creo que debería encomendársele ese nuevo servicio a las Universidades para que den pensiones a los alumnos oficiales y Licenciados.

Hay que ensayar a ver cómo hacen la elección y cuáles son sus relaciones con los muchachos.

Si el Ministro de Hacienda tolera el aumento, podrá consignarse en el Capítulo de Universidades, con el epígrafe:

«Para pensiones en el extranjero a estudiantes de las diversas Facultades... 180.000»

Luego un Decreto organizaría el servicio, y Vd. podría anticipar en las Cortes que se daría plena libertad a las Facultades para hacer la designación de pensionados y que se daría cierta intervención a los estudiantes.

Eso llevaría cierto fermento a la Universidad.

La Junta seguiría con el mismo sistema que ahora tiene, recogiendo gente de toda España para formar el personal, y apretando más en preparación de maestros, pensiones en ingeniería, agricultura, y de todas las carreras indistintamente.

No nos dirán así que queremos monopolio.

Me parece que será de buen efecto aunque el Ministro de Hacienda o la Comisión lo rechacen luego.

20 SÁnchez Ron, J. M. (2006), «En defensa de la JAE. La política científica de José Castillejo», Boletín de la Institución Libre de Enseñanza, II Época, nº 63-64, 67-96, p. 74. 
Además expresará la aspiración a que, como dice Cajal, no se haga esta obra gota a $\operatorname{gota}^{21}$.

\section{CAJAL, EDUCADOR}

Como es sabido, el término educar procede del latín educare, verbo que está emparentado con ducere, que significa «conducir», y «educere», que equivale a «sacar afuera» o «criar». De ahí que en el campo semántico de «educar» se encuentren, entre otras, las nociones de: a) dirigir, encaminar y doctrinar; b) desarrollar las facultades intelectuales y morales del joven por medio de preceptos, ejercicios, o ejemplos; c) perfeccionar y aficionar los sentidos; d) enseñar los buenos usos de urbanidad y cortesía. Y es así que la filóloga María Moliner en su Diccionario del uso del español señale como palabras afines a educar a las siguientes: adiestrar, afinar, amaestrar, civilizar, conformar, corregir, criar, dirigir, disciplinar, documentar, domesticar, ejercitar, enseñar, formar, guiar, preparar, pulir, reprender, tutelar.

Pues bien, prestando atención a la dimensión pública del quehacer de Cajal, tan importante para entender en su totalidad su significación como la personalidad científica más importante de la cultura española en su edad de plata, podemos comprobar cómo las preocupaciones pedagógicas de Cajal atravesaron toda su carrera investigadora. De esa manera su «persona científica» se modeló mediante un ajuste mutuo entre el investigador y sus públicos, de forma análoga a lo que ocurrió en otros casos de insignes científicos, como Darwin ${ }^{22}$.

Por esa razón no ha de extrañar que el tema elegido para su discurso de ingreso en la Real Academia de Ciencias Exactas, Físicas y Naturales el 5 de diciembre de 1897 fuese sobre los Fundamentos racionales y condiciones técnicas de la investigación biológica. ${ }^{23}$ Como él mismo explicó ese día a sus colegas académicos, su pretensión, propia de sus preocupaciones pedagógicas y de su voluntad de convertirse en un educador de los jóvenes «experimentalistas», era la de exponer algunas de las reglas que debían guiar a los biólogos en sus trabajos de observación y experimentación con el afán de «prestar al-

21 CAstillejo, D. (1998), Los intelectuales reformadores de la España contemporánea. Epistolarios de José Castillejo y de Manuel Gómez Moreno. II. El espíritu de una época, 1910-1912. Madrid, Editorial Castalia, pp. 792-793.

22 Browne, J. (2003), "Charles Darwin as a Celebrity», Science in Context, 16. 1, 175-194.

23 Una reproducción de ese discurso en REAl ACADEMIA DE CIENCIAS EXACTAS, Físicas Y NATURAles, (2002): Horizontes culturales. Historia de la ciencia española. Santiago Ramón y Cajal, Madrid, Espasa, pp. 27-49. 
gún servicio a cuantos intentan ensayar sus fuerzas en las investigaciones biológicas; pues con frecuencia hemos visto estudiantes, ganosos de distinguirse y de hacer algo en el terreno experimental, abandonar el laboratorio, desalentados por la falta de un guía que les señalara los errores y obstáculos que deben evitar, la educación técnica que necesitan recibir, y hasta la disciplina moral indispensable para poder abordar, con alguna esperanza de buen éxito, la exploración de la Naturaleza viva».

Fue ese interés por convertirse en un guía de quienes estaban apostando por convertir la «moral de la ciencia» en una moral colectiva dominante en la sociedad española que transitaba del siglo XIX al XX la que le llevó a organizar ese discurso en torno a tres ideas-fuerza: las cualidades morales que debe poseer el investigador, los recursos y métodos que deben caracterizar una buena investigación, y la conveniencia de que los jóvenes investigadores, a los que Cajal quería educar, sustituyesen «los afeites retóricos», que según él eran una plaga desastrosa de la España de su época y «causa muy poderosa de nuestro atraso científico», por una cultura de la precisión, basada en «una severa disciplina de la atención».

Como es sabido, ese discurso académico se convirtió meses después en un libro titulado Reglas y consejos sobre investigación biológica gracias al patrocinio de uno de sus admiradores, el médico hispanocubano Enrique Lluria (1863-1925), uno de los representantes del maridaje del pensamiento evolucionista con ideas socialistas en aquella sociedad española finisecular. No es cuestión de detenerse ahora en los aspectos coyunturales de esa obra, como en su famoso Post-scriptum, redactado al hilo de la conmoción que supuso «el desastre de 1898», añadido que Cajal retiraría en las siguientes ediciones, sino de resaltar que ese texto no sólo es una de las obras más significativas del regeneracionismo científico que surgió en aquella época, sino también uno de sus más importantes éxitos editoriales, pues en vida de su autor tuvo seis ediciones (1897, 1899, 1913, 1916 — aquí añadió Cajal el subtítulo de Los tónicos de la voluntad-, 1920 y 1923) ${ }^{24}$. A lo largo de ese período fue introduciendo modificaciones diversas sobre el núcleo originario del libro, que no era otro, como ya hemos señalado, que su discurso de ingreso en la Real Academia de Ciencias Exactas, Físicas y Naturales. En ellas se perciben sus preocupaciones y obsesiones como pedagogo o educador, como se revela, por

24 Más detalles sobre la recepción de esa obra en el público de habla hispana, y en públicos de otros países en LOPEZ-OCON, L., estudio introductorio a RAMÓN Y CAJAL, S. (2005), Los tónicos de la voluntad. Reglas y consejos sobre investigación científica, Madrid, Gadir, pp. 5-16. 
ejemplo, en el hecho de que dedique el capítulo IX a explicar por qué el investigador tiene que ser también un maestro, y de qué manera - saliendo de su torre de marfil - ha de promover vocaciones científicas. Así como sucede en tantas otras partes de su obra literaria, cabe rastrear en esas páginas referencias autobiográficas, como cuándo expone sus ideas acerca de las características que ha de tener un buen profesor. Pero también se trasluce en ellas al pedagogo en acción que fue Cajal, preocupado por analizar los signos subjetivos y objetivos que permitan hacer un diagnóstico sicológico con el que detectar a los jóvenes talentos susceptibles de alcanzar éxitos científicos. Entre tales signos destaca la «sagacidad para rastrear filones ricos» y la posesión de un «penetrante y seguro sentido crítico», así como «el poder transformador de la imitación» que surge de la convivencia con el maestro.

Los tónicos de la voluntad revelan, pues, que a lo largo de su vida Cajal se tomó muy en serio la complementariedad de su labor científica y su trabajo docente encaminado a formar noveles investigadores. Y así Cajal tuvo una férrea determinación para convertirse en un gran investigador, pero también mostró una persistente voluntad en enseñar a investigar, pues «no se enseña bien sino lo que se hace». Algún testimonio tenemos sobre la calidad de la enseñanza de Cajal, como el de su alumno Gregorio Marañón, para quien las dotes pedagógicas de su maestro radicaban en la claridad de su exposición verbal, nada retórica ni elocuente, y en su dominio del dibujo didáctico, «que no sólo requiere una aptitud artística, sino el difícil sentido de construir, con arte, el esquema que todo lo aclara» $»^{25}$.

Las dotes pedagógicas de Cajal no solo se revelaban en las aulas de las diversas universidades españolas en las que dio clases, como las de Zaragoza, Valencia, Barcelona y Madrid, sino también en sus laboratorios, según testimonios de sus discípulos más próximos ${ }^{26}$, y en su producción literaria, con la que ganó fama de ser uno de los mejores cultivadores de la prosa didáctica en la España de su época, según sostuvo el historiador y crítico literario Melchor Fernández Almagro27.

Sus primeros trabajos de divulgación científica, publicados entre 1883 y 1885 en diversas publicaciones, como la revista zaragozana La Clínica, y

25 PÉrez Gutiérrez, Francisco (1997), La juventud de Marañón, Madrid, Editorial Trotta, p. 123.

26 Ver al respecto Tello, F., (1952), A la memoria de Don Santiago Ramón y Cajal en el primer centenario de su nacimiento, Madrid, Instituto de Biología y Sueroterapia.

27 Fernandez Almagro, M., «La obra literaria de Cajal», en Anexo documental de RAMÓN Y CAJAL, S. (2005), pp. 331-337. 
firmados a veces bajo el seudónimo del Doctor Bacteria, editados no hace mucho por Angel Merchán ${ }^{28}$, demuestran su afán de instruir a los legos en el desarrollo de las ciencias biológicas, transmitiéndoles los conocimientos y las emociones que iba adquiriendo en su trabajo experimental, como se aprecia en sus trabajos titulados «Las maravillas de la Histología», «La máquina de la vida. Estudios populares de Anatomía y Fisiología celulares» o «El más sencillo y seguro de los métodos de coloración de los microbios». La primera parte de Recuerdos de mi vida, que publicó en 1901 con el título de Mi infancia y juventud, cabe verla, a su vez, como un vivo relato de su formación, en la que contó con la compleja orientación de su padre, a quien él terminaría reconociendo como su primer maestro. Y sus otras obras literarias, desde Charlas de café hasta El mundo visto a los ochenta años, también están plagadas de referencias y preocupaciones pedagógicas.

Pero donde se manifiesta claramente el hecho de que Cajal aprovechó su obra literaria como una herramienta educativa fue en el cuento «El hombre natural y el hombre artificial», uno de los apólogos o narraciones seudofilosóficas o seudocientíficas que, escrito hacia 1885 o 1886, es decir, en su etapa valenciana, publicó en 1905 en su libro Cuentos de vacaciones. El valor literario de ese texto es menor, pero es muy expresivo de la «moral» cajaliana, atenta siempre a apreciar la cultura del esfuerzo y la capacidad que tiene el hombre de modelar su propio cerebro observando y estudiando la naturaleza, como le sucede al personaje central del cuento Jaime Miralta. Ese ingeniero y director de una acreditada fábrica de aparatos eléctricos, que tuvo que emigrar a Paris para desplegar en esa ciudad todos sus talentos creativos, se había formado gracias a la eficaz labor pedagógica de un maestro - don Enrique Fernández-, quien en un oscuro valle pirenaico condujo a su alumno «a la contemplación directa de la realidad, guiándole por el mismo camino recorrido por la evolución histórica de la ciencia».

Los coetáneos de Cajal fueron conscientes de la valía como pedagogo de Cajal. Y así, en el homenaje que le hizo la revista Clínica y Laboratorio en un número especial con motivo de la concesión del premio Nobel, Sebastián Recasens, catedrático de Obstetricia y Ginecología en la Facultad de Medicina de Madrid, consideraba que Cajal podría ser un excelente ministro de Instrucción Pública, pues era de esperar que gracias a su inteligencia y a su capacidad de trabajo contribuiría a «sacudir las rutinarias prácticas actuales» y ayudaría «a levantar la cultura pedagógica de nuestro país a un nivel como el alcanzado por

28 Merchán PÉrez, A., ed., (2001), Santiago Ramón y Cajal. Discurso de doctorado y trabajos de juventud, Madrid, Universidad Europea-CEES Ediciones. 
la neurología ${ }^{29}$. Años después —en 1923- Modesto Bargalló, que entonces era profesor en la Escuela Normal de Guadalajara, ordenó y sistematizó los pensamientos sobre educación de Cajal, esparcidos en Reglas y consejos sobre investigación cientifica, Recuerdos de mi vida y Charlas de café, dado su «indudable valor», publicándolos en las prestigiosas ediciones de La Lectura, e insistiendo a sus lectores en que la vida y la labor de Cajal eran «un esfuerzo viviente de autoeducación», y constituían «un caso normativo» ${ }^{30}$.

Ciertamente, Cajal no llegó a ser ministro de Instrucción Pública como le propuso Moret en la primavera de 1906, pocos meses antes de que le concediesen el premio Nobel, pero dispuso de instrumentos políticos y científicos para dejar su huella como pedagogo en la cultura científica española. Así, en compañía de cualificados institucionistas, como José Castillejo, contribuyó desde la presidencia de la Junta para Ampliación de Estudios e Investigaciones Científicas a la reconstrucción del sistema científico español, haciendo posible que un país de endeble tradición científica, caracterizada por sus altibajos y discontinuidades, pasase en un lapsus de un cuarto de siglo de importador a exportador de ciencia, gracias a lo que cabe denominar «la cajalización» de España. En efecto, cabe usar este neologismo para caracterizar al singular proceso mediante el cual se diseminó por muchos laboratorios y lugares de la ciencia españoles una cultura de la precisión y una afición por el trabajo experimental que permitieron la realización de investigaciones punteras y ciencia original en diversos campos del conocimiento, aunque de manera limitada.

Dar cuenta de algunos de los rasgos de ese proceso de «cajalización» impulsado por Cajal desde la presidencia de la JAE es el objetivo de las siguientes páginas, en las que insistiré en el valor ejemplarizante que tuvo la práctica científica y educativa de Cajal en el resto de la comunidad científica española.

\section{CAJAL, PRESIDENTE DE LA JAE}

Cajal fue, sin lugar a dudas, un activo promotor del sistema español de ciencia y tecnología durante su larga y fecunda presidencia de la Junta para

29 RECASENS, S. (1906), «Cajal, político», en Clínica y Laboratorio, 2, pp. 309-311.

30 ver al respecto BARGALló, M. (1923): Los pensamientos de Cajal sobre la educación, Madrid, La Lectura y (1923), «Cajal y sus ideas sobre educación», en Revista de Escuelas Normales, Educación y enseñanza, 1, pp. 2-5. Sobre la labor educativa de Modesto Bargalló ver Bernal MARTínez, J. M. (2001), Renovación pedagógica y enseñanza de las ciencias. Medio siglo de propuestas y experiencias escolares (1882-1936), Madrid, Biblioteca Nueva. 
Ampliación de Estudios e Investigaciones Científicas, institución de la que no fue una mera figura decorativa. El grado de implicación de Cajal en el día a día de la JAE aún no ha sido bien analizado, pero su compromiso con esa institución fue firme. Sabemos que presidía asiduamente las reuniones de su equipo directivo, que tenían lugar una vez al mes. En ellas trabajó por convertir el fomento de la ciencia en un asunto de Estado, y en favorecer un gran pacto entre las grandes fuerzas políticas de la Restauración para promocionar esa actividad cultural.

Estudiosos de ese período del desarrollo de la ciencia española, como el historiador Thomas F. Glick ${ }^{31}$, han considerado que a lo largo del primer cuarto del siglo XX surgió en la sociedad española un discurso civil en materias científicas. Es decir una elite dividida, muy polarizada ideológicamente, pactó poner en suspenso en esa época, de mutuo acuerdo y en determinadas áreas, el hábito de hacer que todas las ideas sirviesen para la confrontación ideológica. Cajal, desde la presidencia de la JAE, contribuyó indudablemente a la consolidación de ese «discurso civil», instando a que todas las decisiones adoptadas durante los primeros años de funcionamiento de esa institución se tomasen por consenso, buscando la unanimidad, a pesar de que en su junta directiva había representantes de diversas corrientes ideológicas y políticas propias de una sociedad pluralista. Así el redactor anónimo de una de las Memorias de la JAE diría al respecto: «Aunque formada por hombres de las más diversas tendencias científicas, políticas, sociales y religiosas, la Junta ha continuado sin interrupción una obra de concordia que trata de implantar reformas trascendentales en que han de estar conformes todos los españoles de buena fe y se inspira en un sentido de escrupulosa tolerancia y de respeto a todas las opiniones. Así merece notarse el hecho de que, fundada en 1907, haya vivido esta Corporación trece años habiendo tomado todos sus acuerdos por unanimidad, sin que las discusiones en que se aportan datos hayan terminado nunca en lucha, votación o disentimiento». Y concluía esta reflexión afirmando «el señor Ramón y Cajal ha presidido asiduamente las reuniones que suelen tener lugar una vez al mes», con lo que estaba planteando implícitamente que esa unanimidad se había logrado, en gran medida, gracias a la autoridad moral de Cajal ${ }^{32}$.

31 Glick, Thomas F., (1986), Einstein y los españoles. Ciencia y sociedad en la España de entreguerras, Madrid, Alianza Universidad.

32 MEMORIA correspondiente a los años 1918 y 1919, (1920), Madrid, Junta para Ampliación de Estudios e Investigaciones Científicas, p. 10. 
La influencia de Cajal en el transcurrir de la JAE fue pues notoria en múltiples aspectos. Ciudadano cuidadoso del manejo de los fondos públicos impuso, por ejemplo, la rendición de cuentas permanente. Año a año en las Memorias de la JAE podemos observar cómo sus responsables, con Cajal a la cabeza, hacían uso de los recursos procedentes de los Presupuestos del Estado o de donaciones particulares.

Pero, sobre todo, su huella, a mi modo de ver, es bien visible en las tres líneas de acción fundamentales que definieron el impulso educativo de la estrategia reformista de ese organismo público de investigación. Esas acciones estratégicas impulsadas por el Cajal educador fueron: la política de envío de pensionados al extranjero con la que se intentó renovar las estructuras académicas y administrativas del Estado español; la creación de centros de investigación científica desde los que los pensionados debían ayudar a reconstruir y modernizar el Estado mediante la práctica de la «moral de la ciencia», y el impulso de instituciones de carácter educativo, como la Residencia de Estudiantes, o el Instituto-Escuela.

En efecto, Cajal y el equipo directivo de la JAE pusieron un particular empeño en proporcionar a la elite de la juventud universitaria los medios para que se formasen en los grandes focos de producción científica de Europa y América. De ahí que desde su momento fundacional se considerase que el objetivo fundamental de la JAE era enviar pensionados al extranjero, hasta el punto de que Cajal en sus escritos denominó a la JAE como Junta de Pensiones y de Estudios. En sus inicios lo que hizo la JAE fue escoger a los jóvenes más aptos que presentaron sus solicitudes y enviarlos al exterior, con el riesgo de que se perdiesen esfuerzos y recursos por una insuficiente preparación. Paulatinamente, a medida que crecieron las posibilidades de una preparación más adecuada por parte de los solicitantes, las exigencias para conceder una pensión, cuya cuantía y duración variaban en cada caso, se hicieron mayores, aunque en términos generales las condiciones solicitadas para pedir pensión no eran rígidas, ni estrechas. Así se aprecia, por ejemplo, en la convocatoria firmada por Santiago Ramón y Cajal, como presidente de la JAE, en Madrid a 29 de diciembre de 1911, y que apareció en la Gaceta de 9 de enero de 1912, para conceder las pensiones en el extranjero durante ese año ${ }^{33}$. Pero dado que siempre fueron más las solicitudes que las concesiones otorgadas (en la convocatoria de 1910 se presentaron 359 solicitudes -151 de profesores de los establecimientos de enseñanza dependientes del Ministerio de Instrucción

33 MEMORIA correspondiente a los años 1912 y 1913 (1914), Madrid, Junta para Ampliación de Estudios e Investigaciones Científicas, pp. 29-33. 
pública y Bellas Artes, y 208 de no profesores- y se concedieron 70; en la de 1911 fueron 455 las solicitudes - 160 de profesores y 295 de no profesores-y se otorgaron 110$)^{34}$ hubo que establecer mecanismos de selección estrictos para acertar en la elección de los pensionados. En primer lugar se hacía una selección eliminatoria para dejar fuera a aquellos que no ofreciesen garantías de competencia y seriedad. Y luego, entre los admisibles, se establecía un orden de preferencia, combinando las condiciones individuales de los candidatos y la naturaleza de los asuntos que se pretendían estudiar con las necesidades del país, y buscando una representación proporcional de los diversos estudios y disciplinas científicas ${ }^{35}$.

Como presidente de la JAE Cajal fue el firmante de las sucesivas convocatorias de pensiones para el extranjero que promovió esa institución. Esa tarea debió de preocuparle, de modo que en diversos momentos de su producción literaria hizo valoraciones de diverso orden sobre ella.

Así dedicó parte del capítulo IX de Los tónicos de la voluntad, como es conocido el libro que originariamente tituló Reglas y consejos sobre la investigación biológica, y que fue uno de sus pocos éxitos editoriales como ya se ha señalado, a hacer un balance de la política de pensiones de la JAE en sus primeros quince años de existencia, dado que la edición definitiva de esa obra la publicó en 1923. Ahí Cajal presentó los logros obtenidos con la política científica de la JAE considerando que se habían recogido «cosechas estimables», de modo que «en la nueva generación el tipo mental del maestro declamador y meramente comentarista disminuye visiblemente, y de día en día aumenta el número de revistas científicas nacionales, de laboratorios y seminarios de investigación y de entusiastas profesores entregados a pesquisas originales». No obstante, consideró que el éxito alcanzado era modesto ya que «avanzamos a paso de tortuga, cuando necesitaríamos velocidades planetarias». Esa situación se debía a tres razones:

$\left.1^{a}\right)$ A la escasez de las pensiones. Para Cajal el escaso centenar de pensionados enviados en el último año era cantidad irrisoria teniendo en cuenta que «nuestro país necesita ser reformado radicalmente de alto a bajo,

34 MEMORIA correspondiente a los años 1910 y 1911 (1912): Madrid, Junta para Ampliación de Estudios e Investigaciones Científicas, pp. 20. 27.

35 Ver al respecto una carta de José Castillejo, fechada en Madrid a 8 de marzo de 1913, a un corresponsal desconocido, citada por GAMERO MERINO, Carmela (1988): «José Castillejo y la Junta: Pensiones en el extranjero», en SANCHEZ RoN, J.M., coord., 1907-1987. La Junta para Ampliación de Estudios e Investigaciones Científicas 80 años después, Madrid, CSIC, vol. II, 29-46, p. 35 y MEMORIA JAE (1912), p. 18. 
hostigando y estimulando al amodorrado cuerpo social hasta la entraña misma». Proponía por tanto para lograr ese objetivo el envío de «cientos y acaso miles de pensionados, legiones de jóvenes decididos a arrancar a Europa el secreto de su grandeza y a infundir un nuevo espíritu en todas nuestras relajadas instituciones docentes y administrativas»".

$2^{a}$ ) A la escasez del tiempo de pensión. A diferencia de Italia, y de otros Estados, donde las pensiones en el extranjero duraban tres años, en el caso español duraban por término medio un año y medio, lo que consideraba Cajal insuficiente, ya que exceptuando el caso de los profesores habituados a la investigación «que visitan los laboratorios extranjeros con la mira de dominar un nuevo método de estudio o de profundizar, al lado del sabio ilustre, algún tema especialísimo» el resto de las pensiones debían prolongarse a los tres años, dada la deficiente preparación técnica y la falta de conocimiento de idiomas de la inmensa mayoría de los doctores y licenciados que optaban a las pensiones.

3) a la escasa edad e insuficiente preparación técnica de los candidatos. Cajal estimaba que se corría «grave riesgo de perder tiempo y dinero enviando al extranjero mozos de veinte a veinticuatro años, ignorantes de sí mismos y sin gustos ni vocación bien definidos», poco formados debido a una defectuosa organización universitaria que se nutría «de mozalbetes irreflexivos, sin formación mental suficiente y casi totalmente desprovistos de conocimientos sólidos en Matemáticas, Física, Química, Historia Natural, Lenguas Vivas y Filosofía», según habían constatado observadores extranjeros conocedores de la organización docente española y críticos de ese sistema educativo como el pedagogo gallego Eloy André, autor del libro La mentalidad alemana ${ }^{36}$, que Cajal recomienda vivamente a sus lectores. De ahí que se mostrase partidario de rechazar como pensionados «a todos los intonsos doctores y licenciados menores de veinticinco años, sin vocación consolidada ni preparación técnica elemental suficiente» ${ }^{37}$.

Para superar en parte esas deficiencias constatadas en el funcionamiento del sistema de pensiones establecido por la JAE el equipo dirigente de esta

36 ANDRÉ, E. L. (1914), La mentalidad alemana. Ensayo de explicación genética del espíritu alemán contemporáneo, Madrid, Daniel Jorro, editor.

37 RAMÓN Y CAJAL, S. (2005), Los tónicos de la voluntad. Reglas y consejos sobre investigación científica, Madrid, Gadir, edición de Leoncio López-Ocón., pp. 240-244. 
institución trazó una estrategia destinada a solventarlas. Por un lado intentó coordinar la obra de las pensiones en el extranjero con la actividad científica y docente que se hacía en España. De modo que a su regreso los pensionados se encontrasen con medios para continuar sus estudios e investigaciones y preparasen a su vez a futuros pensionados. Por esta razón los artífices de la JAE decidieron crear en 1910 una serie de instituciones, de carácter provisional y estructura flexible, en las que confluyesen parte de los primeros pensionados con otros investigadores que estaban esparcidos en diferentes centros de trabajo. Así, un Real Decreto de 18 de marzo de ese año creó un Centro de estudios históricos, y por otro Real Decreto de 27 de mayo de 1910 se constituyó un Instituto nacional de ciencias físico-naturales. Poco después una Real orden de 8 de Junio sentó las bases para una Asociación de laboratorios con el fin de aprovechar los aparatos e instrumentos científicos dispersos en diferentes Centros del Estado.

Esas nuevas instituciones fueron el espacio donde se concentraron profesores, pensionados que habían regresado del extranjero, jóvenes que se preparaban para concursar a una pensión y otros investigadores interesados en abordar problemas científicos diversos. De hecho con el paso del tiempo el Centro de Estudios Históricos se convertiría en un lugar señero del cultivo de las ciencias humanas, y en sede de una potente escuela de filología, creada por Ramón Menéndez Pidal y sus discípulos principales como Américo Castro y Tomás Navarro Tomás ${ }^{38}$. Por su parte el Instituto nacional de ciencias físico-naturales agrupó al Museo de Ciencias Naturales, con sus laboratorios marítimos de Santander y las Baleares, el Museo de Antropología, el Jardín Botánico y el Laboratorio de investigaciones biológicas de Ramón y Cajal, instituciones ya establecidas, a las que se añadieron un Laboratorio de investigaciones físicas, y la Estación alpina de Biología, que se estableció en la Sierra de Guadarrama.

Cajal fue en cierta medida el coordinador de las actividades científicas de todos esos centros de investigación pues el objetivo fundamental de los promotores de la JAE fue «reunir en una colaboración intensa elementos antes dispersos». Y desde la presidencia de la JAE trasladó a la red de laboratorios y de centros de investigación de ese organismo el programa de valores científicos y virtudes cívicas que había puesto en marcha desde su Laboratorio de

38 Un balance y una reflexión sobre la historiografía existente acerca de esa institución en LOPEZ-OCON, L. (1999), «El Centro de Estudios Históricos: un lugar de la memoria», en Boletín de la Institución Libre de Enseñanza, 34-35, pp. 27-48. Una reciente visión panorámica en Lopez Sanchez, J.M., (2006), Heterodoxos españoles. El Centro de Estudios Históricos, 1910-1936, Madrid, Marcial Pons Historia. 
investigaciones biológicas, consistente en alentar el cultivo de la excelencia científica, promocionando la internacionalización de la ciencia española mediante la publicación en las mejores revistas del mundo, y alentando a rendir cuentas permanentemente de los gastos generados en los laboratorios combinando el cultivo de ciencia de calidad con la apertura de los laboratorios a la sociedad para evitar que los científicos se ensimismasen en sus rutinas.

De hecho el Laboratorio de investigaciones biológicas de Cajal, creado por el gobierno de Francisco Silvela tras una intensa campaña de prensa después de que el Congreso internacional de Medicina celebrado en Paris en 1900 concediese a Cajal el premio internacional Moscú, marcó la pauta del funcionamiento científico del conglomerado de laboratorios de la JAE. Desde sus inicios Cajal obtuvo importantes éxitos en el seno de ese laboratorio, particularmente el hallazgo en el segundo semestre de 1903 del método de nitrato de plata, una fórmula de impregnación susceptible de provocar coloraciones intensas, y perfectamente transparentes, de la urdimbre de las células nerviosas para determinar si esos filamentos podían considerarse vías intracelulares, especialmente diferenciadas para la propagación del impulso nervioso ${ }^{39}$, con vistas a convencer definitivamente a sus rivales reticularistas de la fuerza de sus ideas y argumentos en la controversia que tenía entablada Cajal sobre la estructura y función del sistema nervioso. Esos éxitos galvanizaron las energías de un selecto grupo de discípulos, como Francisco Tello, consolidaron internacionalmente su fama de excelente investigador experimental que le llevaría a la obtención del premio Nobel, y sentaron las bases de la creación de una potente escuela histológica española ${ }^{40}$.

No ha de extrañar por tanto que Cajal no sólo fuese uno de los organizadores de la política científica de la JAE, sino que su ascendiente científico sea palpable en todos los niveles del funcionamiento de la institución desde su momento fundacional. Alentó a investigadores de su círculo, que luego formarían parte de su escuela, a hacer un «tour» formativo por los principales laboratorios del mundo en el campo de su especialidad. Así Gonzalo Rodríguez Lafora (1886-1971), quien con el paso de los años se convertirá en un relevante neurólogo y siquiatra, director desde 1916 del Laboratorio de Fisiología y Anatomía de los Centros Nerviosos, será uno de los integrantes de la primera pro-

39 Las condiciones en que se produjo ese hallazgo y sus consecuencias en su programa de investigaciones se describen en RAMÓN Y CAJAL, S. (1981), caps. XIX y XX, particularmente pp. 242-250.

40 GonzÁlez Santander, R. (1986-2001), La Escuela Histológica Española, 4 vols., Universidad de Alcalá; AGUiRRE DE VIANI, C. (2002), Cajal y su escuela, Salamanca, Junta de Castilla y León. 
moción de pensionados de la JAE, integrada por gente que luego serían figuras relevantes de la ciencia, la cultura y la política española como Eduardo Hernández Pacheco, Enrique Moles, Julio Rey Pastor, Ramón Carande, José Ortega y Gasset, Manuel Azaña, Julián Besteiro, entre otros. Lafora aprovechó bien las oportunidades que se le ofrecieron: trabajó en Berlin sobre Anatomía del sistema nervioso con importantes profesores, y luego en Munich sobre Histopatología de las enfermedades mentales en el laboratorio de Alzheimer, donde hizo cuatrocientas preparaciones microscópicas, trasladándose posteriormente a Paris, Roma e Inglaterra. Fruto de esas investigaciones fueron una serie de publicaciones que dio a conocer en revistas españolas, norteamericanas y alemanas como el Monatschrift für Psychiatrie und Neurologie, de Berlin ${ }^{41}$.

Contribuyó asimismo para que investigadores como Nicolás Achúcarro (1880-1918), quien había adquirido una muy buena formación neuropatológica y psiquiátrica trabajando en Munich con Kraepelin y Alzheimer, y había organizado el servicio de anatomía patológica del manicomio de Washington, se abriese un espacio en el sistema investigador español. Y así le dio facilidades para que abriese una línea de investigación de histopatología del sistema nervioso en un pequeño laboratorio provisional, dependiente del que dirigía Cajal. En él iniciaría a un grupo de alumnos en los métodos de investigación histológica, en el sistema nervioso y enfermo. Cajal le convenció asimismo para que en el marco de los cursos de ampliación que auspiciaba la JAE impartiese durante una hora a la semana unas «Lecciones de histopatología de la corteza cerebral, con demostraciones», cuya finalidad era la de exponer mediante el apoyo de la proyección micrográfica y del microscopio los elementos histológicos y las alteraciones del sistema nervioso en enfermedades nerviosas y mentales ${ }^{42}$.

Y también prestó sus materiales de trabajo e instrumentos para que cursos impartidos por investigadores de la JAE destinados a difundir la cultura de la precisión en el seno de la sociedad española fuesen más lucidos y provechosos a los alumnos. Así Domingo de Orueta organizó un curso sobre «Investigaciones y ensayos prácticos sobre fotografía microscópica» para, entre otros objetivos, dar reglas y consejos prácticos «encaminados a facilitar el manejo del microscopio en sus líneas generales y a sentar un criterio sobre la mayor o menor eficacia de los aparatos y procedimientos más usuales». Se hicieron entonces experimentos en el único banco de óptica de que se disponía, que

41 MEMORIA correspondiente a los años 1910 y 1911 (1912), Madrid, Junta para Ampliación de Estudios e Investigaciones Científicas, pp. 87-88.

42 MEMORIA correspondiente a los años 1910 y 1911 (1912), Madrid, Junta para Ampliación de Estudios e Investigaciones Científicas, pp. 153, 175. 
era el modelo grande de la casa Zeiss de Jena, acompañado de la cámara horizontal de dicha casa. Pues bien ambos instrumentos fueron facilitados por Ramón y Cajal a Domingo de Orueta ${ }^{43}$.

Finalmente cabe señalar que el prestigio que Cajal fue acumulando en el transcurso de los años con su programa de investigaciones, con su labor educativa y con sus dotes de gestor científico sirvió para que su nombre sirviese de imán para captar recursos adicionales para la Junta para Ampliación de Estudios e Investigaciones Científicas, que esta usó para poner en marcha nuevas líneas de investigación. Así a finales de la década de 1920 la Institución Cultural Española de Buenos Aires decidió financiar en Madrid la denominada cátedra Cajal con recursos procedentes de una suscripción entre la colonia española en Argentina cuya finalidad era contratar profesores extranjeros que impartiesen cursos y dirigiesen trabajos de investigación en los laboratorios de la JAE. Esa cátedra, financiada inicialmente con 50.000 ptas, se inauguró oficialmente en octubre de 1928 y la JAE decidió que tuviese como objetivo prioritario durante tres cursos consecutivos la promoción de trabajos sobre rayos $\mathrm{X}$ y estructura de los cristales, línea de investigación que dirigía el físico Julio Palacios. Para tal fin se decidió invitar al profesor Paul Scherrer de la prestigiosa Escuela politécnica superior de Zurich para que desarrollase en el Laboratorio de investigaciones físicas de la JAE en Madrid un ambicioso programa de trabajos prácticos. Ahora bien a fines de 1928 solo se habían gastado 10.067, 78 ptas del total de la cantidad aportada desde Argentina: 3.600 ptas para remunerar las actividades de Paul Scherrer y Julio Palacios, y 6.467, 78 ptas para la adquisición de diversos instrumentos y aparatos como bombas de difusión, tubo de Hadding, goniómetro, cámaras para rayos $\mathrm{X}^{44}$.

Hasta el fin de sus días, producido el miércoles 17 de octubre de 1934, Cajal no sólo estuvo científicamente activo, sino hondamente preocupado por el devenir de la sociedad española, y por los resultados de su labor científica, pedagógica, y política. Así lo puede comprobar quien lea la última de sus obras El mundo visto a los ochenta años, desde la que oteó su entorno y dio sus adioses. En ella también hizo balance de su quehacer como gestor científico y educador, constatando que gracias a la Junta de Pensiones y Ampliación de Estudios como él denominó a la JAE se había facilitado «la formación de una grey de ingenieros, abogados, humanistas, médicos, físicos, químicos, naturalistas y hasta filósofos, impregnados de los secretos de la técnica y de

43 Ibid., (1912), p. 167.

44 MEMORIA correspondiente a los cursos 1926-1927 y 1927-1928, Madrid, Junta para Ampliación de Estudios e Investigaciones Científicas, 1929, pp. 184-185, 368, 404. 
los métodos inquisitivos ultrapirenaicos o ultramarinos». Pero también constataba que «en nuestra prometedora ascensión cultural no todas las disciplinas científicas y sus aplicaciones marchan isocrónicamente. En ciertas actividades (matemáticas, estudios históricos, histología, ciencias naturales, etc) comenzamos a hombrearnos con los extraños, aunque sin igualarlos todavía; pero en otros, verbi gratia, la ingeniería, la zootecnia, la bacteriología, la botánica práctica, la astronomía, la química, la física, y sobre todo el arte de la invención industrial, vamos a la zaga.... $\rangle^{45}$.

Hasta tal punto la voluntad pedagógica de Cajal seguía viva al final de su vida que su contribución al Archivo de la Palabra, el gran proyecto puesto en marcha por el fonetista del Centro de Estudios Históricos Tomás Navarro Tomás durante el bienio 1931-1933 para registrar la palabra de las personalidades españolas e hispanoamericanas más representativas en el campo de las ciencias, de las letras y de las artes, fuese el de «Pensamientos de tendencia educativa». En ellos resumió parte de su ideario educativo, y su confianza en la fuerza ejemplarizante del investigador docente para orientar la labor de los jóvenes científicos, pues en su opinión «misión trascendental del educador es desarrollar alas en los que tienen manos y manos en los que tienen alas. Sólo trabajando se enseña a trabajar. Como decía Cisneros, Fray Ejemplo es el mejor predicador» ${ }^{46}$.

Así pues podríamos afirmar para finalizar estas reflexiones que con su esfuerzo perseverante, tanto de carácter científico, como educativo, y político, Cajal y sus compañeros gestores de la JAE consiguieron que el sistema español de ciencia y tecnología diese un salto de calidad, pasando de la periferia a la semi-periferia del sistema científico mundial. En esa situación aun nos mantenemos, décadas después de su fallecimiento y de la desaparición de la JAE, ya que las pérdidas generadas en el sistema científico-técnico español por el estallido de la guerra civil fueron de tal magnitud, que aún no han podido ser subsanadas, como sucedió por ejemplo con el debilitamiento de la Escuela española de histología, al tener que exiliarse varios de los discípulos de Santiago Ramón y Cajal.

45 RAmón y CAJal, S. (2000), Obras selectas, Madrid, Espasa Calpe, Austral Summa, pp. 738-743.

46 La transcripción completa de las reflexiones de Cajal en Archivo de la Palabra, Publicaciones de la Residencia de Estudiantes, CSIC, 1990, p. 28. La transcripción completa de las reflexiones de Cajal en Archivo de la Palabra, Publicaciones de la Residencia de Estudiantes, CSIC, 1990, p. 28, Se reproducen en el anexo que se incluye al final de este texto. 


\section{ANEXO DOCUMENTAL \\ Intervención de Santiago Ramón y Cajal en el Archivo de la Palabra Pensamientos de tendencia educativa}

Si hay algo en nosotros verdaderamente divino es la voluntad. Por ella afirmamos la personalidad, templamos el carácter, desafiamos la adversidad, reconstruimos el cerebro y nos superamos diariamente.

Te quejas de las censuras de tus maestros, émulos y adversarios cuando debieras agradecerlas. Sus golpes no te hieren, te esculpen. Con pocas excepciones, todo joven dotado de acusada y fuerte personalidad reacciona contra las exageraciones doctrinales o sentimentales de padres y maestros adoptando el tono o colorido moral complementario. El tumulto de la vida social suele obrar sobre las cabezas humanas débiles como el río sobre un cristal de cuarzo. Arrastrado y golpeado por la corriente conviértese al fin en vulgar canto rodado. Quien desee conservar incólumes las brillantes facetas de su espíritu recójase prontamente en el remanso de la soledad, tan propicio a la actividad creadora.

Natural y loable es el ansia de reputación. Importa empero que el maestro discierna los dos principales tipos de ambiciosos: los que codician la fama como fin y los que la persiguen como medio. Cultívese de preferencia a los primeros. Misión trascendental del educador es desarrollar alas en los que tienen manos y manos en los que tienen alas. Sólo trabajando se enseña a trabajar. Como decía Cisneros, Fray Ejemplo es el mejor predicador.

Nos gustan los libros que relatan las hazañas que hubiéramos deseado realizar, es decir un programa de vida noble y bella frustrado por el aciago destino.

Suele crecer la planta según la dimensión de la maceta. El talento aldeano confinado en su rincón difícilmente alcanzará su pleno florecimiento.

La naturaleza nos ha otorgado dotación limitada de células cerebrales. He aquí un capital, grande o pequeño, que nadie puede aumentar, ya que la neurona es incapaz de multiplicarse. Pero si se nos ha negado la posibilidad de acrecentar el caudal celular, se nos ha otorgado en cambio el inestimable privilegio de modelar, ramificar y complicar las expansiones de esos elementos, como si dijéramos de los hilos telegráficos del pensamiento, para combinar casi hasta el infinito las asociaciones reflejas y las creaciones ideales. Aprovechémonos de esta preciosa prerrogativa durante la juventud y la edad viril, porque el protoplasma neuronal parece endurecerse como el mortero con el transcurso del tiempo y no hay nada más infecundo y aun nocivo que una cabeza incapaz de aprender y corregirse.

Existen dos variedades humanas, de valor harto desigual: el hombre rebañego, modelado por la tradición y la rutina y el hombre nuevo forjado por autorreflexión. Esta variable mental merece exclusivamente el nombre de individuo, porque sólo él es capaz de aportar algo al acervo común del progreso. Las cabezas sencillas y sugestionables reproducen el tipo humano ancestral, orientadas hacia el pasado desdeñan el futuro. Son empero necesarias, ya que forman la reserva evolutiva de la raza, donde laten en potencia, aguardando su hora, los genios del porvenir. 


\section{Ideal de la ciencia}

Puesto que vivimos en pleno misterio, luchando contra las fuerzas desconocidas, tratemos en lo posible de esclarecerlo. Concluida nuestra labor seremos olvidados, como la semilla en el surco. Pero algo nos consolará el considerar que nuestros remotos descendientes nos deberán dar un poco de su dicha y que gracias a nuestro esfuerzo el mundo resultará algo más agradable e inteligible. Una severa autocrítica constituye el más precioso don del pensador. Nada de embriagarse con el propio vino, bueno o malo. No imitemos la credulidad confiada de la gallinácea que incuba con la misma formalidad un huevo fecundo que un huevo de mármol.

Sobre la primacía de la teoría sobre la práctica y viceversa se han gastado mares de tinta. Hoy, al contrario de otras épocas, prevalece la exageración practicista con lo que se obtienen buenos obreros, pero rutinarios y mediocres maestros. Se olvida demasiado que el problema docente es un problema de equilibrio mental. 